\title{
Validity and repeatability of the Vicorder apparatus: a comparison with the SphygmoCor device
}

\author{
Stacey S Hickson ${ }^{1}$, Mark Butlin ${ }^{2,3}$, Jeremy Broad ${ }^{1}$, Alberto P Avolio ${ }^{2}$, Ian B Wilkinson ${ }^{1}$ \\ and Carmel M McEniery ${ }^{1}$
}

Aortic stiffness, an independent predictor of cardiovascular risk and all-cause mortality, can be estimated non-invasively by measuring carotid to femoral (aortic) pulse wave velocity (aPWV). The Vicorder device has been developed to measure aPWV with little operator training in a non-intrusive manner. The aim of this study was to assess the repeatability of aPWV measured with the Vicorder device and to compare aPWV values with those obtained using the SphygmoCor system. Vicorder and SphygmoCor aPWV was assessed in 122 subjects ( $53 \pm 18$ years, 46 male) using both the manufacturers' and a standardized approach. Vicorder aPWV measurement proved to be highly repeatable (within-subject coefficient of variation $2.8 \%$ ). Transit time differed significantly between the two devices (mean difference $22 \pm 9 \mathrm{~ms}, P<0.001$ ), independent of the different algorithms used to calculate transit time. However, aPWV was similar between the two devices (mean difference $0.31 \pm 1.54 \mathrm{~m} \mathrm{~s}^{-1}, P<0.001$ ) though with an inherent bias toward lower Vicorder aPWV values at high values of SphygmoCor aPWV. Bias was reduced by subtracting the additional femoral artery segment measured by the Vicorder device, also bringing the measure of transit time in closer agreement to SphygmoCor values (mean difference $5 \pm 9 \mathrm{~ms}, P<0.001$ ). Transit time values significantly differed between the two devices and the Vicorder device reported lower aPWV values at higher SphygmoCor values of aPWV. This difference in transit time and inherent bias was reduced when adjustment for the additional femoral artery segment measured by the Vicorder device was made. Hypertension Research (2009) 32, 1079-1085; doi:10.1038/hr.2009.154; published online 25 September 2009

Keywords: arterial stiffness; pulse wave velocity; SphygmoCor; Vicorder

\section{INTRODUCTION}

Aortic stiffness is an independent predictor of cardiovascular risk and cardiovascular outcome in a variety of patient groups. ${ }^{1-5}$ Arterial stiffness can be assessed by a variety of methods, but aortic pulse wave velocity (aPWV) is the current 'gold-standard' measure. ${ }^{6} \mathrm{aPWV}$ itself is an independent predictor of cardiovascular risk ${ }^{1,5}$ and is related to the elastic properties of the arterial wall. Pulse wave velocity (PWV) is inversely related to arterial distensibility and directly related to Young's elastic modulus. ${ }^{7}$ It is defined as the time taken by a pressure or flow wave to travel a given distance. PWV can be determined from dual, non-invasive recordings of femoral and carotid artery pressure or flow waveforms. The transit time is usually calculated using a foot-to-foot methodology and the distance by measuring along the surface of the body between the recording points with a tape measure or callipers. Some devices such as the SphygmoCor system (AtCor Medical, West Ryde, NSW, Australia) use sequential recordings of the waveform with ECG gating. Others, including the Complior system (Artech Medical, Pantin, France) allow recording of simultaneous waveforms. Although these two devices yield differing values for aPWV, this is mainly due to differences in the in-built algorithms used to calculate the transit time, rather than the methods used to acquire the waveforms. ${ }^{8}$ Indeed, if the SphygmoCor algorithm is applied to the Complior waveforms, comparable estimates of aPWV are obtained. Although both devices are widely used, and provide repeatable estimates of $\mathrm{aPWV},{ }^{9,10}$ they require considerable operator training and are intrusive in that they require exposure and palpation of the femoral artery.

The more recent Vicorder system (Skidmore Medical, Bristol, UK) measures simultaneous pressure waveforms by a volume displacement technique, using blood pressure cuffs placed around the sites of interest. As such, little operator training is required and it is less intrusive. The aim of this study was to assess the comparability and repeatability of aPWV measurements using the Vicorder device, with those made using the SphygmoCor system, as a reference standard. ${ }^{11,12}$

\section{METHODS}

Subjects

Subjects were randomly drawn from the Anglo-Cardiff Collaborative Trial. ${ }^{11}$ Individuals with peripheral vascular disease, or atrial fibrillation, were excluded

${ }^{1}$ Clinical Pharmacology Unit, University of Cambridge, Addenbrooke's Hospital, Cambridge, UK and ${ }^{2}$ The Australian School of Advanced Medicine, Macquarie University, Sydney, Australia

${ }^{3}$ Co-first author.

Correspondence: SS Hickson, Clinical Pharmacology Unit, University of Cambridge, Vascular Research Clinics, ACCI Level 3, Box 110, Addenbrooke's Hospital, Hills Road, Cambridge $\mathrm{CB} 2 \mathrm{OQQ}$, UK.

E-mail: sh529@cam.ac.uk

Received 26 March 2009; revised 20 July 2009; accepted 4 August 2009; published online 25 September 2009 
from the study, as were current cigarette smokers. Approval was obtained from the Local Research Ethics Committee, and written informed consent was obtained from all participants.

\section{Measurement techniques}

The supine aPWV was determined by applanation tonometry using the SphygmoCor device by sequentially recording ECG-gated carotid and femoral artery pressure waveforms as described earlier. ${ }^{10,13}$ A total of $10 \mathrm{~s}$ of highquality waveforms were captured for each reading, and the intersecting tangents algorithm was applied to determine the foot-to-foot transit time using the integrated software and the $R$ wave as a reference frame. Path length was calculated as directed by the manufacturer: the distance from the femoral measurement site to the suprasternal notch (notch-femoral) minus the distance from the carotid site to the suprasternal notch (notch-carotid).

Measurements were obtained using the Vicorder device by placing a $100 \mathrm{~mm}$ wide blood pressure cuff around the upper thigh to measure the femoral pulse and a $30 \mathrm{~mm}$ partial cuff around the neck at the level of the carotid artery. The cuffs were each inflated to $60 \mathrm{~mm} \mathrm{Hg}$, and high-quality waveforms were recorded simultaneously for $3 \mathrm{~s}$ with the subject in the supine position, using a volume displacement method. The foot-to-foot transit time was determined using an in-built cross-correlation algorithm centered around the peak of the second derivative of pressure. Path length was defined as the distance from the suprasternal notch to the top of the thigh cuff as indicated by the manufacturer. Typical carotid and femoral waveforms recorded with both the SphygmoCor and Vicorder devices are represented in Figure 1.

\section{Study 1: Comparison of Vicorder and SphygmoCor aPWV measures}

A total of 122 subjects were studied. After 15 min of supine rest in a quiet temperature-controlled environment, brachial artery blood pressure and heart rate were measured using an oscillometric device (Omron 705CP, Kyoto, Japan). The aPWV was then measured using the Vicorder and SphygmoCor systems sequentially, in random order, as detailed above. All measurements were made in duplicate and the average value used in subsequent analyses.

Earlier studies ${ }^{8}$ have confirmed the importance of the algorithm used to determine the foot of the pressure waveform and the subsequent transit time. To study the influence of the different algorithms on aPWV values, pressure waveforms recorded using the Vicorder device were then re-sampled at $128 \mathrm{~Hz}$ and analyzed using SphygmoCor software and the intersecting tangents algorithm.

\section{Study 2: Repeatability of vicorder aPWV measurements}

After completion of Study 1, the repeatability of Vicorder aPWV measurements was assessed in a subset of 33 subjects. Two additional recordings of aPWV were made using the Vicorder apparatus approximately $5 \mathrm{~min}$ apart by the same operator.
Study 3: Identification of the point of detection of the femoral pulse The value of aPWV derived from any device is largely dependent on accurate determination of the distance between the sites of measurement. The femoral cuff used by the Vicorder device is $100 \mathrm{~mm}$ wide, which, given an average notch-femoral length of $450 \mathrm{~mm}$ and aPWV of $8 \mathrm{~m} \mathrm{~s}^{-1}$, may yield an error in aPWV estimation of $22 \%$ if the actual point of detection of the pressure waveform was at the bottom rather than the top of the cuff. Therefore, a further experiment was conducted in 10 subjects to define the precise point of waveform detection in the femoral cuff. After $15 \mathrm{~min}$ supine rest, aPWV was measured using the Vicorder in the usual manner and the transit time noted. The $100 \mathrm{~mm}$ femoral cuff was then removed and a smaller cuff (width $50 \mathrm{~mm}$ ) was placed around the thigh, centered in turn, and in random order, over the position of the top, middle, and bottom of the larger cuff. For each position, aPWV was again measured and transit time recorded. All measurements were made in triplicate and the mean was used for analysis. Transit times to each cuff position were compared to determine the precise point in the larger cuff in which the femoral pressure waveform was being recorded.

\section{Study 4: Correcting for the femoral segment of aortic PWV}

If an additional length of artery of fixed stiffness is included in the aPWV measurement, this will cause measured aPWV to deviate from true aPWV, particularly at high levels of aPWV. ${ }^{13,14}$ The Vicorder device includes a longer segment of the femoral artery than the SphygmoCor device because the Vicorder cuff position is distal to the palpable point of the femoral artery (Figure 2), and earlier data indicate that femoral PWV increases to a lesser degree than aPWV with age. ${ }^{15}$ Therefore, mathematically removing the additional femoral segment from the Vicorder aPWV measure may correct for any inherent bias at high aPWV.

To determine the PWV in the femoral segment, a $100 \mathrm{~mm}$ wide blood pressure cuff was placed around the upper part of the thigh, as proximal to the

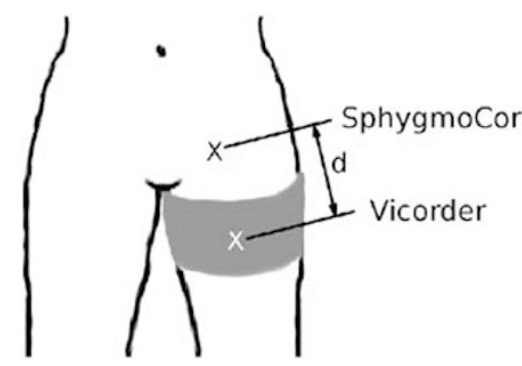

Figure 2 The diagram shows the palpable point of the femoral pulse as used with the SphygmoCor device (black X) and the effective point of detection of the femoral pulse in the Vicorder thigh cuff (white $X$ ). The two points are separated by a distance (d). This distance of femoral artery is included in aPWV measurement by the Vicorder device, but not the SphygmoCor device.
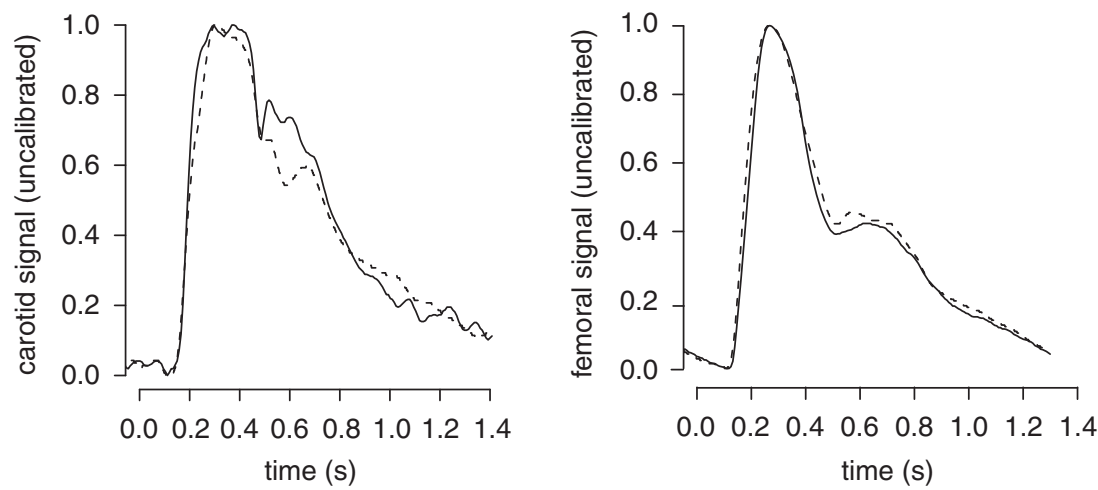

Figure $1 \mathrm{~A}$ carotid and femoral artery pulse waveform as recorded in a single subject (26 year old female) using the SphygmoCor (solid line) and Vicorder (dashed line) devices. Despite the different techniques used to detect the pulse and the small differences in the site of detection, the recorded waveforms show good agreement on visual inspection, with some degree of variation in the diastolic portion of the carotid pulse. 
groin as possible, and a smaller cuff (width $50 \mathrm{~mm}$ ) was placed just superior to the patella. The Vicorder apparatus was then used to measure transit time, the distance between the centers of the two cuffs was determined, and PWV was calculated. Triplicate measurements were made in 20 individuals.

\section{Statistics}

All values are expressed as mean \pm one standard deviation. Agreement between SphygmoCor and Vicorder values were analyzed with Student's paired $t$-tests with further affirmation by Bland-Altman plots. ${ }^{16}$ Linear regression by the method of ordinary least squares was used to define the correlation between SphygmoCor and Vicorder values, with goodness of fit expressed by Pearson's

Table 1 Subject demographics and hemodynamics

\begin{tabular}{|c|c|c|}
\hline Parameter & Study $1(\mathrm{n}=122)$ & Study $2(\mathrm{n}=33)$ \\
\hline Age (years) & $53 \pm 18$ & $45 \pm 15$ \\
\hline Male/female $(n)$ & $46 / 76$ & $11 / 22$ \\
\hline Height (m) & $1.7 \pm 0.1$ & $1.7 \pm 0.1$ \\
\hline Weight (kg) & $71 \pm 12$ & $69 \pm 10$ \\
\hline Body mass index $\left(\mathrm{kg} \mathrm{m}^{-2}\right)$ & $25 \pm 4$ & $24 \pm 3$ \\
\hline Systolic blood pressure $(\mathrm{mm} \mathrm{Hg})$ & $127 \pm 19$ & $118 \pm 11$ \\
\hline Diastolic blood pressure $(\mathrm{mm} \mathrm{Hg})$ & $73 \pm 10$ & $71 \pm 7$ \\
\hline Mean blood pressure $(\mathrm{mm} \mathrm{Hg})$ & $91 \pm 11$ & $87 \pm 8$ \\
\hline Pulse blood pressure (mm Hg) & $55 \pm 16$ & $48 \pm 8$ \\
\hline Heart rate (beats $\min ^{-1}$ ) & $65 \pm 11$ & $71 \pm 8$ \\
\hline
\end{tabular}

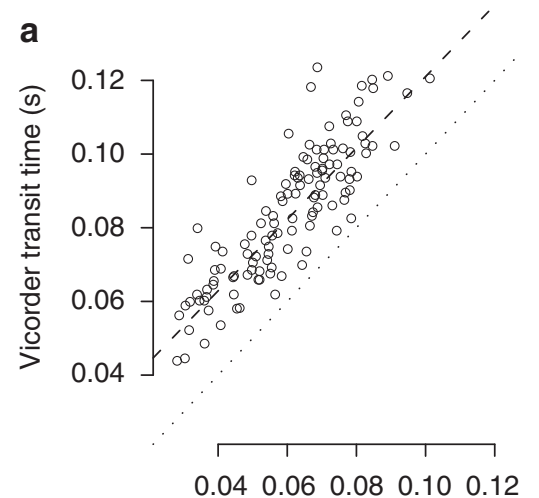

Sphygmocor transit time (s)

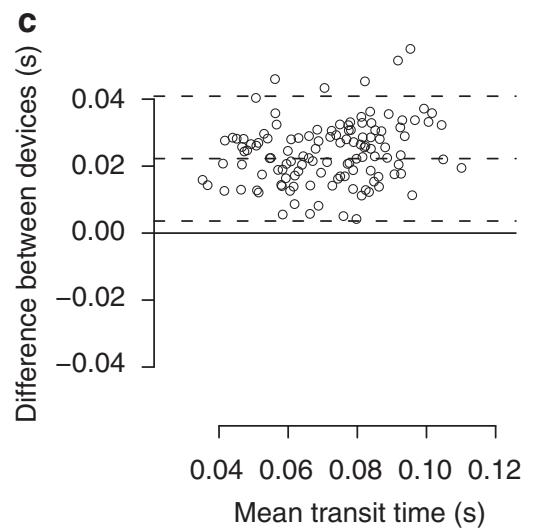

correlation coefficient $(r)$. Repeatability of Vicorder aPWV measurements was assessed by Bland-Altman analysis. ${ }^{16}$ All analysis was conducted using the statistical package, $R .^{17}$

\section{RESULTS}

Study 1: Comparison of Vicorder and SphygmoCor aPWV measures

The basic demographics of the 122 subjects included in Study 1 are presented in Table 1. There were more women than men (76 and 46, respectively), but a wide range of ages (19-92 years).

Carotid to femoral transit times were significantly different between the Vicorder and SphygmoCor devices $(83 \pm 18$ and $60 \pm 16 \mathrm{~ms}$, respectively, mean difference $22 \pm 9 \mathrm{~ms}, P<0.001)$. However, there was overall good linear agreement in transit time between devices (slope $=0.97, r=0.86, P<0.001$; Figure $3 \mathrm{a}$ and $\mathrm{c}$ ). In spite of poor agreement between transit times, mean aPWV was in relatively close agreement (Vicorder $8.3 \pm 2.2 \mathrm{~m} \mathrm{~s}^{-1}$, SphygmoCor $8.6 \pm 2.9 \mathrm{~m} \mathrm{~s}^{-1}$, difference $0.3 \pm 1.5, P=0.14$ ). However, although there was reasonable linear correlation between the two sets of recordings $(r=0.85$, $P<0.001$; Table 2), there was evidence of inherent bias in the data with a relative underestimate of aPWV by the Vicorder device compared with aPWV determined by the SphygmoCor system at aPWV values over $10 \mathrm{~m} \mathrm{~s}^{-1}$ (Figure $3 \mathrm{~b}$ and d). This was evidenced by a marginally better fit of quadratic regression $(r=0.86)$ than linear regression $(r=0.85)$. There was good agreement between Vicorder and SphygmoCor values of aPWV within each age decade (Figure 4),

\section{b}

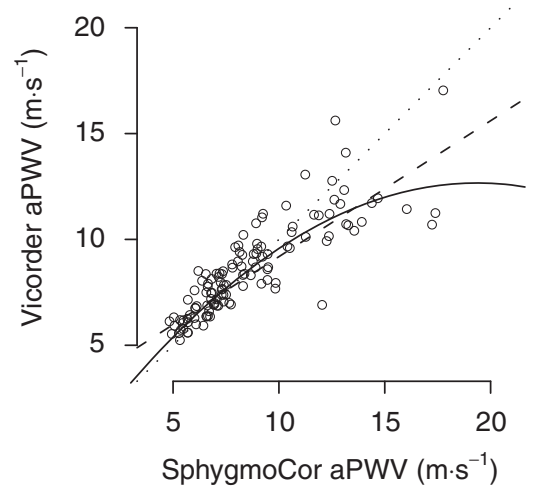

d

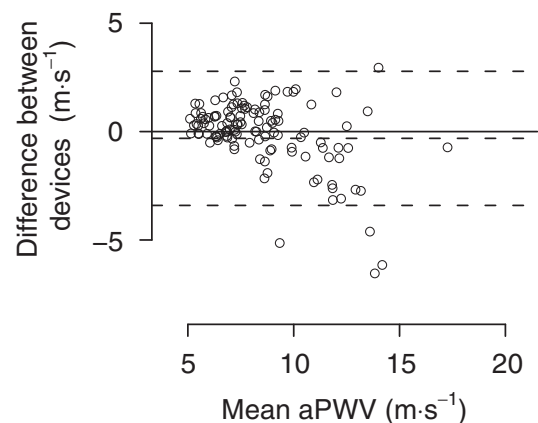

Figure 3 SphygmoCor and Vicorder carotid to femoral transit time (a) and aPWV (b) calculated with distances measured by manufacturer specifications. The dotted line represents unity, the dashed line linear regression, and the solid line quadratic regression. Table 2 reports $r$ and $P$-values. Plots (c) and (d) show the corresponding measurement agreement in Bland-Altman plots. 
Table 2 Mean and regression statistics for aPWV measures plotted against SphygmoCor aPWV

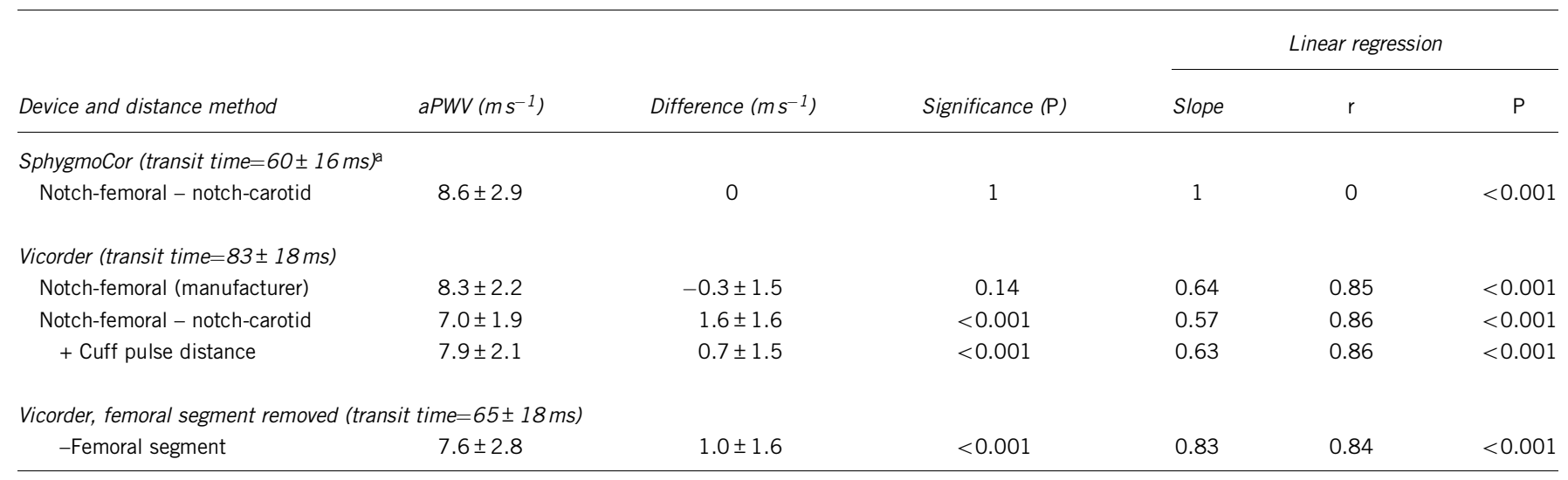

Abbreviation: aPWV, aortic pulse wave velocity.

aReference value.

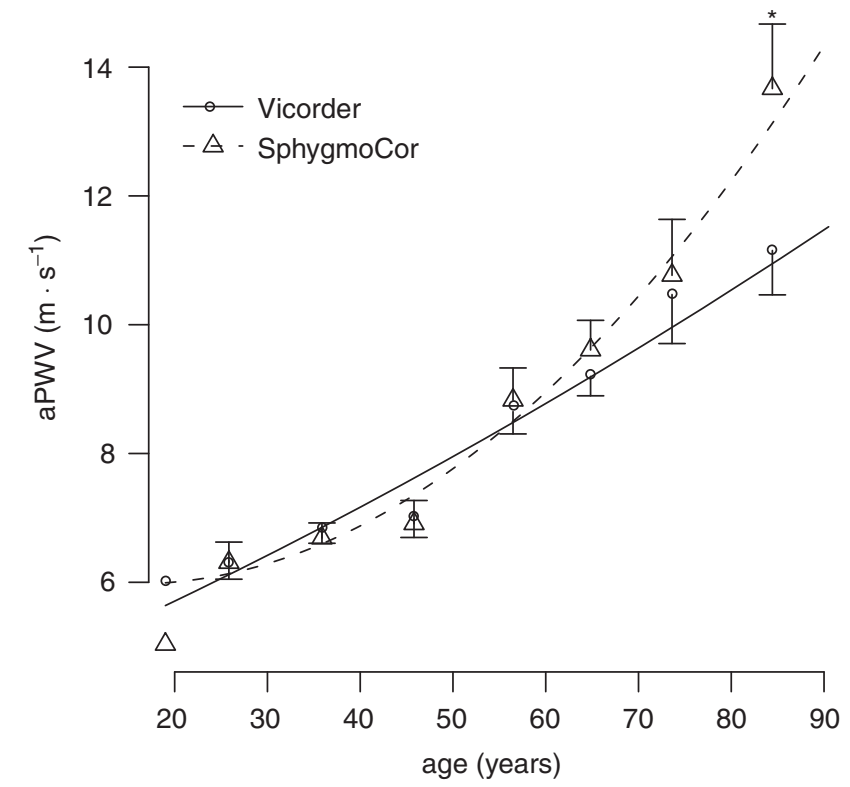

Figure 4 The relationship between Vicorder and SphygmoCor aPWV values and age. Differences in aPWV were only observed in the highest age decade $(P<0.05$, Student's paired $t$-test). The regression curve based on the original Vicorder measurements was aPWV $=0.0001 \times$ age $^{2}+0.06 \times$ age +4.4 $(r=0.68)$. Regression on SphygmoCor values resulted in the relationship of aPWV $=0.001 \times$ age $^{2}-0.05 \times$ age $+6.3(r=0.71)$, closer to values observed by McEniery et al., ${ }^{11}$ also using the SphygmoCor device.

with a statistical difference detected only in the 80-90 years age bracket (Vicorder $11.2 \pm 0.7 \mathrm{~m} \mathrm{~s}^{-1}$, SphygmoCor $13.7 \pm 1.0 \mathrm{~m} \mathrm{~s}^{-1}$, $P<0.05)$.

The close agreement of aPWV recorded by the two devices was surprising, given the marked difference in transit times, and the different protocol used to determine path length: notch-femoral minus notch-carotid for SphygmoCor $(477 \pm 47 \mathrm{~mm})$ compared with simply notch-femoral for the Vicorder $(649 \pm 58 \mathrm{~mm}$, $P<0.001)$. To adjust for the difference in measurement technique, Vicorder aPWV was recalculated using the method adopted by the SphygmoCor manufacturer, as it provided closer approximation to true arterial path length. When the same technique of path length measurement was used (notch-femoral distance minus notch-carotid distance), there was a significant difference between aPWV values given by the two devices (Table 2). The inherent bias in data at higher values of aPWV remained (Figure 5a).

To assess the influence of the different pressure wave foot-finding algorithms of the two devices on the measured transit time, a subset of 42 Vicorder carotid-femoral waveforms were re-analyzed by processing the Vicorder recorded waveforms with SphygmoCor software. There was no difference between the original Vicorder transit times and those calculated using the SphygmoCor software (Vicorder transit time $87 \pm 19 \mathrm{~ms}$, SphygmoCor software transit time $85 \pm 19 \mathrm{~ms}$, $r=0.94, P<0.001$, mean difference of $1 \pm 7 \mathrm{~ms}, P=0.15)$. Therefore, the algorithms do not contribute to the observed difference in transit times between devices.

\section{Study 2: Repeatability of Vicorder aPWV measurements}

Repeatability was assessed in 33 subjects. aPWV values for repeatability analysis ranged from 5.5 to $10.5 \mathrm{~m} \mathrm{~s}^{-1}$ with a mean difference between paired aPWV values of $0.01 \pm 0.29 \mathrm{~m} \mathrm{~s}^{-1} \quad(P=0.92)$ and within-subject coefficient of variation of $2.8 \%$.

Study 3: Identification of the point of detection of the femoral pulse The mean transit time when aPWV was measured using the large Vicorder thigh cuff was $96 \pm 8 \mathrm{~ms}$. Transit times when the smaller thigh cuff was placed in the top, middle, and bottom positions of the large cuff were $87 \pm 6,94 \pm 7$, and $101 \pm 8 \mathrm{~ms}$, respectively. Therefore, the effective position of the pressure waveform measurement was calculated as $65 \pm 27 \mathrm{~mm}$ from the top of the large thigh cuff (designated ' $\mathrm{X}$ ' in Figure 2). In view of this finding, the original data collected in Study 1 were then re-analyzed adjusting for the extra distance to the pulse detection point. This yielded a closer approximation to the SphygmoCor aPWV when the same path length measurement technique was applied (Table 2), but the inherent bias at higher values of aPWV still remained (Figure 5b).

\section{Study 4: Correcting for the femoral segment of aortic PWV}

Femoral PWV was measured in 20 subjects ( 9 male, age $48 \pm 19$ years) using the Vicorder device. The mean femoral PWV was $9.4 \pm 3.2 \mathrm{~m} \mathrm{~s}^{-1}$. Using this mean value and the mean distance between the femoral pulse detected using SphygmoCor to that detected by the Vicorder (162 $\pm 35 \mathrm{~mm}$, SphygmoCor to Vicorder femoral distance, Figure 2), mean transit time within this segment was calculated to be $17 \mathrm{~ms}$. The Vicorder carotid to femoral transit time was then corrected for the additional femoral segment by subtraction of the calculated femoral transit time $(17 \mathrm{~ms})$ and mean SphygmoCor to Vicorder 

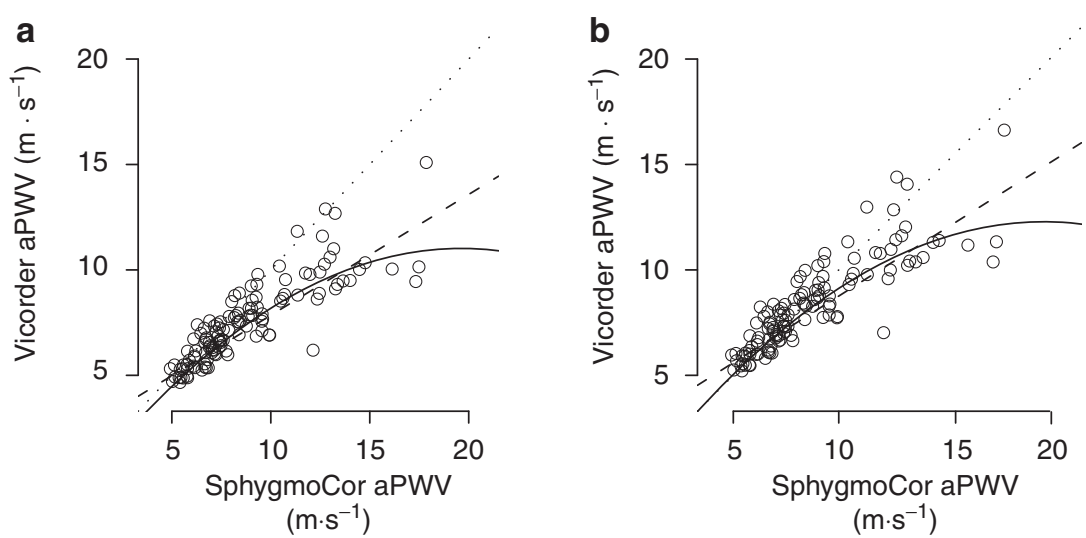

Figure 5 Vicorder aPWV with corrected distances plotted against SphygmoCor aPWV. (a) Vicorder aPWV with the notch-carotid distance subtracted. (b) Vicorder aPWV with both the notch-carotid distance subtracted, and the distance to the pulse detection point in the femoral cuff added. The dotted line represents unity, the dashed line linear regression, and the solid line quadratic regression. Table 2 reports $r$ and $P$-values.

distance of $162 \mathrm{~mm}$ (Equation (1)).

$$
\mathrm{PWV}_{\text {adjusted }}=\frac{\text { notch-femoral distance }- \text { notch-carotid distance }+65 \mathrm{~mm}-162 \mathrm{~mm}}{\text { transit time }}
$$

The adjusted Vicorder transit time and the original SphygmoCor transit time had a mean difference of $5 \pm 9 \mathrm{~ms}(P<0.001)$, closer than the unadjusted Vicorder transit time values. Transit time between devices remained highly correlated $(r=0.86, P<0.001$, Figure 6a). Similarly, adjusted Vicorder aPWV was closer to the original SphygmoCor aPWV than Vicorder aPWV measured using a standardized distance measurement, although the values were still significantly different (mean difference $1.0 \pm 1.6 \mathrm{~m} \mathrm{~s}^{-1}, P<0.001$, linear regression $r=0.84, P<0.001$, Figure $6 \mathrm{~b}$ ). The modeled subtraction of the femoral segment reduced the inherent bias at higher values of aPWV, as evidenced by improved Pearson's correlation coefficient and nearer approximation of unity slope by linear regression (Table 2).

\section{DISCUSSION}

At present, there are a number of commercially available devices designed for aPWV measurement, including PulsePen (DiaTecne, Milan, Italy), Complior (Artech Medical), SphygmoCor (AtCor Medical) and the newly developed Vicorder (Skidmore Medical). The Complior system has been shown to both underestimate ${ }^{8}$ and overestimate ${ }^{18,19}$ aPWV compared with the SphygmoCor system. Aortic PWV measured using PulsePen was overestimated compared with Complior measurements, ${ }^{20}$ though both devices were strongly correlated with aPWV measured by simultaneously acquired carotid and femoral pressure waveforms using two Millar tonometers. ${ }^{20,21}$ The Vicorder device has not been assessed in relation to other aPWV measuring devices. Therefore, the aim of this study was to determine the repeatability of aPWV measured with the Vicorder device and to compare aPWV measurements with those of the SphygmoCor system across a wide range of aPWV values by means of assessing subjects across a wide range of age. As a current standard for aPWV measurement does not exist, the SphygmoCor system was chosen as the reference standard in this study. This was chosen over simultaneous, dual tonometric pressure pulse analysis due to the widespread use and proven high repeatability of the SphygmoCor device. ${ }^{10,11}$ The main finding from this study was that transit time values measured by the Vicorder system significantly differed from those recorded using the SphygmoCor system. In spite of this, the highly repeatable Vicorder measures of aPWV were on average highly correlated with SphygmoCor aPWV measures. However, at higher stiffness values, there was evidence of an inherent bias toward lower Vicorder aPWV compared with SphymoCor aPWV values. Modeled adjustment for the additional segment of femoral artery measured by the Vicorder system greatly improved agreement in transit times between the devices and reduced the bias seen at higher stiffness values.

It was unexpected that when used as per the manufacturer's instructions, the Vicorder device gave similar values of aPWV to the SphygmoCor system, given that the path length was measured using a different technique. However, as both the path length and transit time were greater with the Vicorder system, it is not surprising that the values of Vicorder aPWV and SphygmoCor aPWV were in good agreement. When the Vicorder recordings were recalculated using path length measured in the same manner as the SphygmoCor system (subtracting the notch-carotid distance from the notch-femoral distance), the absolute difference in aPWV between Vicorder and SphygmoCor increased, but had no effect on the inherent bias. Further distance correction to include the distance to the effective point of pulse detection within the thigh cuff $(65 \mathrm{~mm}$ from the top of the cuff) not only improved the agreement in aPWV between devices, but also had no effect on the inherent bias.

Systematic bias has been reported in other devices. Millasseau et al. ${ }^{8}$ noted that the Complior device consistently calculated lower PWV values than the SphygmoCor. This bias was attributed to the algorithm used to determine the foot of the pressure waveform. When pressure waveforms recorded with the Complior device were re-analyzed with the intersecting tangents algorithm of the SphygmoCor device, the bias was removed. In this study, re-analysis of pressure waveforms recorded with the Vicorder device using the intersecting tangents algorithm of the SphygmoCor software did not correct for the inherent bias. Therefore, a difference in algorithms used to locate the foot of the pressure waveform did not contribute to the difference in measured transit times observed between the Vicorder and SphygmoCor devices. It may also be possible that the accuracy of transit time measurement is affected by obesity by dampening the transmission of the pulse to the site of measurement. However, the Vicorder device seemed to give un-dampened recordings of both carotid and femoral waveforms in obese subjects, and this is unlikely to explain the bias. 
a

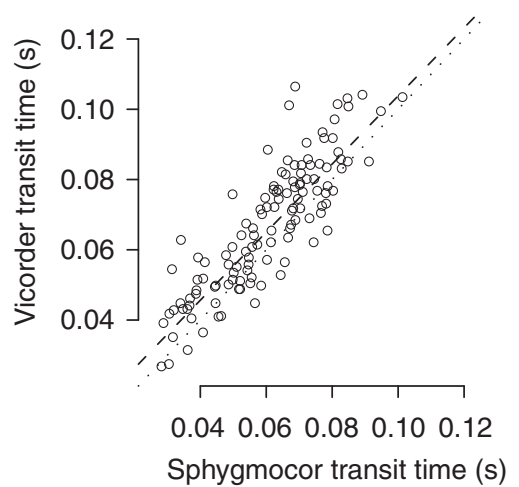

C

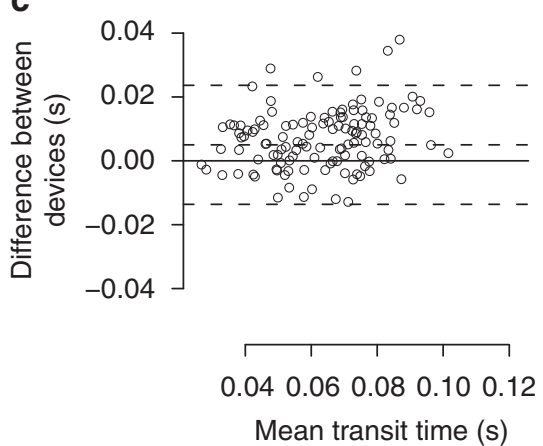

b

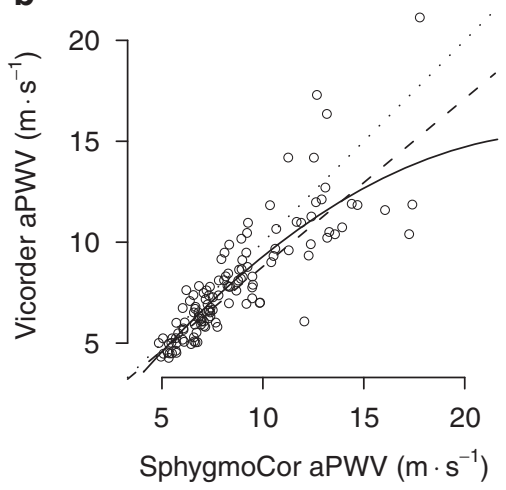

d

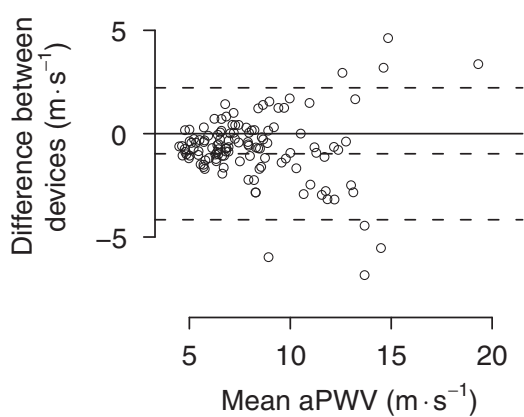

Figure 6 Vicorder carotid to femoral transit time (a) and aPWV (b) as estimated to the site of the SphygmoCor femoral pulse compared with SphygmoCor carotid to femoral measurements. The line of unity is represented by the dotted line, linear regression by the dashed line, and quadratic regression by the solid line. Table 2 reports $r$ and $P$-values. Plots (c) and (d) show the corresponding measurement agreement in Bland-Altman plots.

Another explanation for the inherent bias may be related to the fact that the Vicorder, but not the SphygmoCor device, includes a longer segment of femoral artery (Figure 2). If the stiffness of this additional arterial segment is relatively constant between individuals, it may introduce an inherent bias at high aPWV. Indeed, the observation that peripheral PWV does not vary greatly throughout life ${ }^{14,15}$ supports this hypothesis. Therefore, we modeled a correction by subtracting the additional segment of femoral artery measured by the Vicorder system from the original values. This brought transit time in closer agreement with that calculated by SphygmoCor, though the difference between the systems remained statistically significant. Nonetheless, the inherent bias at values of aPWV over $10 \mathrm{~m} \mathrm{~s}^{-1}$ was greatly reduced. Mean aPWV measures, however, did not improve in agreement between devices. The modeled corrections for the Vicorder device were based on a relatively small number of subjects, and further investigations that include a larger population sample are required.

Bias at high values of aPWV could also be caused by a difference in signal sampling rate between the two devices. A low signal-sampling rate could cause proportionally larger errors in transit time measurement at higher values of aPWV by means of aliasing. The Vicorder device samples and analyzes waveforms at $556 \mathrm{~Hz}$. At a PWV of $14 \mathrm{~m} \mathrm{~s}^{-1}$, over an effective path length of $450 \mathrm{~mm}$, this results in a maximum overestimation of $0.83 \mathrm{~m} \mathrm{~s}^{-1}$ and underestimation of $-0.74 \mathrm{~m} \mathrm{~s}^{-1}$. The SphygmoCor device acquires data at $128 \mathrm{~Hz}$ and analyzes waveforms at an interpolated and re-sampled rate of $1 \mathrm{kHz}$, leading to a maximum overestimation of $0.45 \mathrm{~m} \mathrm{~s}^{-1}$ and underestimation of $-0.42 \mathrm{~m} \mathrm{~s}^{-1}$ at a PWV of $14 \mathrm{~m} \mathrm{~s}^{-1}$. Therefore, this small error cannot explain the inherent bias seen in the current data.
On the basis of the findings of this study, we suggest a standard method of distance calculation for the Vicorder path length that is representative of anatomical arterial path length, consistent with path length measures to date, and similar to that of the SphygmoCor manufacturer's recommendation. The linear distance from suprasternal notch to the top of the thigh cuff and suprasternal notch to the carotid artery should be measured with tape measure or callipers, and the effective path length calculated as defined in equation (2), the additional $65 \mathrm{~mm}$ being the distance to the pulse detection point in the thigh cuff.

$$
\begin{aligned}
& \text { Arterial path length } \\
& \quad=(\text { notch to top of thigh cuff })-(\text { notch to carotid pulse })+65 \mathrm{~mm}
\end{aligned}
$$

In summary, the findings of this study indicate that Vicorder transit time measures are significantly different to those measured by the SphygmoCor device. In spite of this, the highly repeatable Vicorder measures of aPWV are, on average, in good agreement with SphymoCor measures when path length is measured in a standardized manner, with correction for the distance to the pulse detection point in the thigh cuff. However, at higher values of aortic stiffness, the Vicorder system reports lower aPWV values than those of the SphymoCor device. The discrepancy in transit time between the two devices is reduced by adjusting for the additional segment of femoral artery measured by the Vicorder device, which also reduces the inherent bias at higher values of aortic stiffness. However, there is still disagreement at high aPWV, which may be important clinically, given that aPWV is most predictive at values $>\sim 12 \mathrm{~m} \mathrm{~s}^{-1}$. This may limit the clinical 
usefulness of the Vicorder device until a suitable correction is implemented.

\section{CONFLICT OF INTEREST}

The authors declare no conflict of interest.

\section{ACKNOWLEDGEMENTS}

The sources of support are SS Hickson: Cambridge Overseas Trust \& Commonwealth Trust; M Butlin: Australian Research Council Discovery Grant DP0773971; IB Wilkinson; CM McEniery: British Heart Foundation Fellowships; Support from the Biomedical Research Center.

1 Blacher J, Guerin AP, Pannier B, Marchais SJ, Safar ME, London GM. Impact of aortic stiffness on survival in end-stage renal disease. Circulation 1999; 99. 2434-2439.

2 Boutouyrie P, Tropeano Al, Asmar R, Gautier I, Benetos A, Lacolley P, Laurent S. Aortic stiffness is an independent predictor of primary coronary events in hypertensive patients: a longitudinal study. Hypertension 2002; 39: 10-15.

3 Laurent S, Boutouyrie P, Asmar R, Gautier I, Laloux B, Guize L, Ducimetiere P, Benetos A. Aortic stiffness is an independent predictor of all-cause and cardiovascular mortality in hypertensive patients. Hypertension 2001; 37: 1236-1241.

4 Laurent S, Katsahian S, Fassot C, Tropeano AI, Gautier I, Laloux B, Boutouyrie P. Aortic stiffness is an independent predictor of fatal stroke in essential hypertension. Stroke 2003; 34: 1203-1206.

5 Meaume S, Benetos A, Henry OF, Rudnichi A, Safar ME. Aortic pulse wave velocity predicts cardiovascular mortality in subjects $>70$ years of age. Arterioscler Thromb Vasc Biol 2001; 21: 2046-2050.

6 Laurent S, Cockcroft JR, Van Bortel L, Boutouyrie P, Giannattasio C, Hayoz D, Pannier B, Vlachopoulos C, Wilkinson IB, Struijker-Boudier H, on behalf of the European Network for Non-Invasive Investigation of Large Arteries. Abridged version of the expert consensus document on arterial stiffness. Artery Res 2007; 1: 2-12.

7 Nichols WW, O'Rourke MF. McDonald's Blood Flow in Arteries: Theoretical, Experimental and Clinical Principles, 5th edn. Arnold: London, 2005
8 Millasseau SC, Stewart AD, Patel SJ, Redwood SR, Chowienczyk PJ. Evaluation of carotid-femoral pulse wave velocity: influence of timing algorithm and heart rate. Hypertension 2005; 45: 222-226.

9 Ueda I, Tagawa T, Watanabe S, Yamakawa K, Yasu T, Ueda S. Comparability and reproducibility of the carotid-femoral pulse wave velocity measurements using a multielement carotid tonometry sensor. J Hum Hypertens 2008; 22: 699-703.

10 Wilkinson IB, Fuchs SA, Jansen IM, Spratt JC, Murray GD, Cockcroft JR, Webb DJ. Reproducibility of pulse wave velocity and augmentation index measured by pulse wave analysis. J Hypertens 1998; 16 (12 Pt 2): 2079-2084.

11 McEniery CM, Yasmin, Hall IR, Qasem A, Wilkinson IB, Cockcroft JR. Normal vascular aging: differential effects on wave reflection and aortic pulse wave velocity: the AngloCardiff Collaborative Trial (ACCT). J Am Coll Cardiol 2005; 46: 1753-1760.

12 Wojciechowska W, Staessen JA, Nawrot T, Cwynar M, Seidlerova J, Stolarz K, Gasowsk J, Ticha M, Richart T, Thijs L, Grodzicki T, Kawecka-Jaszcz K, Filipovsky J. Reference values in white Europeans for the arterial pulse wave recorded by means of the SphygmoCor device. Hypertens Res 2006; 29: 475-483.

13 Chiu YC, Arand PW, Shroff SG, Feldman T, Carroll JD. Determination of pulse wave velocities with computerized algorithms. Am Heart J 1991; 121: 1460-1470.

14 van der Heijden-Spek JJ, Staessen JA, Fagard RH, Hoeks AP, Boudier HA, van Bortel LM. Effect of age on brachial artery wall properties differs from the aorta and is gender dependent: a population study. Hypertension 2000; 35: 637-642.

15 Avolio AP, Chen SG, Wang RP, Zhang CL, Li MF, O'Rourke MF. Effects of aging on changing arterial compliance and left ventricular load in a northern Chinese urban community. Circulation 1983; 68: 50-58.

16 Bland JM, Altman DG. Statistical methods for assessing agreement between two methods of clinical measurement. Lancet 1986; 1: 307-310.

17 R Developtment Core Team. R: A Language and Environment for Statistical Computing. R Foundation for Statistical Computing, Vienna, Austria, ISBN: 3-900051-07-0, 2008.

18 Baulmann J, Schillings U, Rickert S, Uen S, Dusing R, Illyes M, Cziraki A, Nickering G, Mengden T. A new oscillometric method for assessment of arterial stiffness: comparison with tonometric and piezo-electronic methods. J Hypertens 2008; 26: 523-528.

19 Rajzer MW, Wojciechowska W, Klocek M, Palka I, Brzozowska-Kiszka M, KaweckaJaszcz K. Comparison of aortic pulse wave velocity measured by three techniques: Complior, SphygmoCor and Arteriograph. J Hypertens 2008; 26: 2001-2007.

20 Salvi P, Magnani E, Valbusa F, Agnoletti D, Alecu C, Joly L, Benetos A. Comparative study of methodologies for pulse wave velocity estimation. J Hum Hypertens 2008; 22: 669-677.

21 Salvi P, Lio G, Labat C, Ricci E, Pannier B, Benetos A. Validation of a new non-invasive portable tonometer for determining arterial pressure wave and pulse wave velocity: the PulsePen device. J Hypertens 2004; 22: 2285-2293. 